\title{
ON SYMPLECTIC MANIFOLDS WITH SOME CONTACT PROPERTIES
}

\author{
YAKOV ELIASHBERG
}

\section{Introduction}

We show in this article that a symplectic manifold bounded by the standard contact sphere is, under some additional hypotheses, a ball. This gives a tool for the recognition of nonstandard structures on spheres, and we show here that exotic contact structures on spheres of dimension $>3$ do exist. These examples are of a completely different nature than Bennequin's nonstandard contact structure on $S^{3}$ (see [1]). The new nonstandard structures on high-dimensional spheres are $s$-fillable (see $\S 3$ below); i.e., they bound symplectic manifolds, while Bennequin's nonstandard structure is overtwisted (see [2]) and, therefore, cannot bound a symplectic manifold (see [5], [3]). In the other paper (see [3]) we show that the only $s$-fillable contact structure on $S^{3}$ is the standard one.

During the preparation of the paper the author had many stimulating discussions with $\mathrm{D}$. McDuff. In particular, she proposed that the main Theorem 6.1 be deduced from the Theorem 5.1 on the structure of asymptotically flat symplectic manifolds (which is proved in her paper) instead of giving the similar proof that was originally intended. A. Floer and D. McDuff explained their proof of homological triviality of asymptotically flat symplectic manifolds. The author wants to thank them, as well as T. Januszkiewicz and C. Viterbo, for interesting discussions.

This paper was essentially completed when the author visited the University of Rome "La Sapienza." $\mathrm{He}$ is sincerely grateful to Italian mathematicians and especially to Professors I. Cattaneo-Gasparini, S. Marchiafava, F. Succi, A. Carfagna, and R. Mazzocco for their hospitality and interest.

Received January 11, 1989. Research at the Mathematical Sciences Research Institute supported in part by National Sciences Foundation grant DMS-812079-05. 


\section{Symplectic and contact manifolds: basic definitions}

A symplectic manifold $(W, \omega)$ is a smooth $2 k$-dimensional manifold $W$ with a nondegenerate $\left(\omega^{k} \neq 0\right)$ closed 2-form $\omega$. The form $\omega$ defines an orientation of $W$ (via the volume form $\omega^{n}$ ) and a cohomology class $[\omega] \in H^{2}(W, \mathbb{R})$ which is not zero if $\omega$ is closed.

A contact manifold $(V, \xi)$ is a smooth $(2 k-1)$-dimensional manifold $V$ with a completely nonintegrable codimension 1 tangent plane distribution $\xi$, i.e., a distribution which could be at least locally defined by a 1-form $\alpha$ such that $\alpha \wedge(d \alpha)^{k-1}$ nowhere vanishes. This means that $\left.d \alpha\right|_{\xi}$ is symplectic on $\xi$. Multiplication of the form $\alpha$ by a function causes multiplication of the symplectic structure $\left.d \alpha\right|_{\xi}$ by the same function. Therefore, conformal symplectic class of $\left.d \alpha\right|_{\xi}$ depends only on $\xi$. We will denote it by $C S(\xi)$. If $k$ is even, then the contact structure $\xi$ defines the orientation of the manifold $V$ (because the sign of $\alpha \wedge(d \alpha)^{k-1}$ does not depend, in this case, on the sign of $\alpha$ ). For any $k$ we get an orientation of $V$ by fixing the sign of the conformal symplectic structure $C S(\xi)$.

\section{Symplectic structure dominating a given contact structure}

Let $V$ be a closed manifold, $W=V \times(0,+\infty)=V \times \mathbb{R}_{+}$, and $T$ be the vector field $\partial / \partial t$ on $W$, where $t$ is the projection $W=V \times \mathbb{R}_{+} \rightarrow \mathbb{R}_{+}$on the second factor. We will identify $V$ with the submanifold $V \times 1 \subset W$. Let $\xi$ be a contact structure on $V$ and $\omega$ be a germ of a symplectic structure on $W$ defined near $V=V \times 1 \subset W$. We say that $\omega$ dominates $\xi$ if $\left.\omega\right|_{\xi} \in C S(\xi)$ and if the contact orientation of $V$, which arises by choosing the sign of $C S(\xi)$ to be equal to the sign of $\left.\omega\right|_{\xi}$, together with the vector field $T$ defines the symplectic orientation of the germ of the symplectic manifold $\left.(W, \omega)\right|_{V \times 1}$.

Note that if a contact structure $\xi$ on $V$ can be dominated by a symplectic structure, then $\xi$ and $V$ are orientable. Conversely, any orientable contact structure on an orientable manifold admits an exact dominating form $\omega$ on $W$, the symplectization of $\xi$, which can be written in the form $\omega=d(t \alpha)$ for a 1 -form $\alpha$ defining $\xi$.

Proposition 3.1. Let $\omega$ be an exact form dominating a contact structure $\xi$ on $V$. Then there exists a symplectic structure $\Omega$ on $V \times \mathbb{R}_{+}$, which coincides with the symplectization $\omega_{\xi}$ of $\xi$ on $V \times[C, \infty)$ for a constant $C>1$ and is equivalent to $\omega$ near $V \times 1$. 
Proof. There exists a 1-form $\alpha$ and an exact 2-form $\theta=d \beta$ on $V$ such that $\{\alpha=0\}=\xi,\left.\theta\right|_{\xi}=0$, and $\omega$ is equivalent to the form $\omega^{\prime}=$ $d(t \alpha)+\theta$ near $V \times 1 \subset W$. Let $h: \mathbb{R}_{+} \rightarrow \mathbb{R}_{+}$be a smooth monotone function such that

$$
h(t)= \begin{cases}1, & t \leq 1+\varepsilon \\ 0, & t>C\end{cases}
$$

let $\Omega=d(t \alpha)+d(h \beta)$. It is easy to see that $\Omega$ coincides with $d(t \alpha)$ for $t>C$, coincides with $\omega^{\prime}$ for $t<1+\varepsilon$, and is symplectic if $h^{\prime}(t)$ is sufficiently small. But the last condition can be easily fulfilled if $C$ is large enough.

Any symplectic form $\omega$ admits a tame (see [5]) almost complex structure $J$ which is homotopically unique. Hence we can associate (via a dominating symplectic form) with an orientable contact structure $\xi$ on an orientable manifold $V$ an (almost) complex structure $J(\xi)$ which is defined on the stable tangent bundle $T^{S}(V)$ of $V$.

\section{Symplectic manifolds with contact boundary}

If $V$ is a boundary of a manifold $W$, we can identify a tubular neighborhood of $V$ in $W$ with a neighborhood of $V \times 1$ in $V \times(0,1] \subset V \times \mathbb{R}_{+}$ such that the vector field $T$ corresponds to an outward transversal vector field to $V$ in $W$.

We say that a compact symplectic manifold $(W, \omega)$ is bounded by a contact manifold $(V, \xi)$ if $V=\partial W$ and $\omega$ dominates $\xi$.

A contact manifold $(V, \xi)$ is called $s$-fillable (or symplectically fillable) if it bounds a symplectic manifold.

A symplectic manifold $(W, \omega)$ is said to be with contact type boundary if its boundary $\partial W$ admits a contact structure $\xi$ dominated by $\omega$.

If $\omega$ is exact near $\partial W$, then the last definition is equivalent to the definition of contact type boundary by A. Weinstein [9].

A noncompact symplectic manifold is called asymptotically flat (the term belongs to A. Floer) if it is symplectomorphic to the standard $\mathbb{R}^{2 k}$ outside a compact. The next proposition is an immediate corollary of 3.1 .

Proposition 4.1. Let $(W, \omega)$ be a symplectic manifold bounded by the standard contact sphere. Then it admits a symplectic embedding into an asymptotically flat symplectic manifold. 


\section{The structure of asymptotically flat symplectic manifolds}

Theorem 5.1 (Gromov, McDuff, Floer, Eliashberg). Let $W^{2 k}$ be an asymptotically flat symplectic manifold. If the space $W$ contains no 2dimensional symplectic spheres, then $W$ is diffeomorphic to $\mathbb{R}^{2 k}$.

For the case $k=2$, the theorem was proved in more strong form (symplectomorphism instead of diffeomorphism) by Gromov in [5]. Using Gromov's technique of pseudoholomorphic curves, McDuff and Floer proved independently the homological triviality of $W$ for $k>2$. I completed the proof by the observation that the simply connectedness can also be proved in the same way. The complete proof of the theorem is contained in [6].

If $k=2$, then McDuff proved in [6] that $W$ is symplectomorphic to $\mathbb{C}^{2}$ with a finite number of points blown up even without the assumption about the nonexistence of symplectic 2-spheres. The symplectic structure on $W$ in this case is generated by a Kähler metric which is standard near infinity.

\section{Symplectic manifolds bounded by the standard contact sphere}

The following theorem is an immediate corollary of 4.1 and 5.1.

Theorem 6.1. Let $\left(W^{2 k}, \omega\right)$ be a compact symplectic manifold bounded by the standard contact sphere. Suppose that $W$ contains no 2-dimensional symplectic spheres. Then the manifold $W$ is diffeomorphic to the ball $B^{2 k}$.

If $k=2$, then it is true, without the assumption about 2-dimensional symplectic spheres, that $W$ is diffeomorphic to the connected sum $B^{4} \sharp n \overline{C P^{2}}$ and exceptional 2-spheres can be chosen symplectic.

\section{Nonstandard contact structures on spheres}

Theorem 4.1 gives a tool to distinguish an $s$-fillable contact structure on a sphere from the standard one. Using this observation we will get nonstandard $s$-fillable contact structures on spheres $S^{2 k-1}$ for $k>2$. Nonstandard contact structures on $S^{3}$ were constructed by D. Bennequin [1] and, as already mentioned in the Introduction, have a completely different nature: They are not $s$-fillable (see [3] and [5]).

A contact structure $\xi$ on $S^{2 k-1}=\partial B^{2 k}$ is called homotopically standard if the corresponding complex structure $J(\xi)$ on the stable tangent bundle $T^{s}\left(S^{2 k-1}\right)=\left.T\left(B^{2 k}\right)\right|_{S^{2 k-1}}$ can be extended to the ball $B^{2 k}$ as an almost complex structure. 
Theorem 7.1. For any $k>2$, the sphere $S^{2 k-1}$ admits an s-fillable nonstandard contact structure. If $k$ is odd, then this nonstandard structure can be made homotopically standard.

The theorem immediately follows, in view of 6.1, from Propositions 7.2, 7.3, and 7.4 below. Propositions 7.2 and 7.3 are proved in [4], but the second one is quite simple and was known earlier (see [7]).

Proposition 7.2. Let $W$ be a symplectic manifold and $\varphi: L \rightarrow W$ be a Lagrangian immersion with only transversal double intersection points. Then the image $\varphi(L)$ has an arbitrarily small regular neighborhood with contact type boundary.

Proposition 7.3. Let $W_{1}$ and $W_{2}$ be two symplectic manifolds of the same dimension with contact type boundaries. Then their connected sum $W_{1} \sharp W_{2}$ at boundary points admits a structure of symplectic manifold with contact type boundary.

Proposition 7.4. For any $k>2$, there exists a $(k-1)$-connected, but not contractible, $2 k$-dimensional manifold bounded by a contact sphere. The contact structure on the boundary can be made homotopically standard if $k$ is odd.

Proof. Let $N$ be a tubular neighborhood of the zero section in the cotangent bundle of the sphere $S^{k}$. Then $N$ has a canonical symplectic structure for which the zero section is Lagrangian. Now using a number of copies of $N$, we apply the plumbing construction (see [8]) and for any graph $\Gamma$ we get a manifold $N_{\Gamma}$ which inherits the symplectic structure. The "soul-spheres" of copies of $N$ form an immersed Lagrangian submanifold $L_{\Gamma} \subset N_{\Gamma}$ with transversal double intersection points. Hence we can apply 7.2 and choose a neighborhood of $L_{\Gamma}$ in $N_{\Gamma}$ with the contact type boundary. We will denote this neighborhood also by $N_{\Gamma}$. By Milnor's theorem [8] we can choose for any $k>2$ a graph $\Gamma$, such that $\partial N_{\Gamma}$ is a homotopy sphere. But groups of homotopy spheres are finite and, therefore, there exists an integer $n>0$, such that the boundary of the connected sum (at boundary points) $\sharp n N_{\Gamma}$ of the $n$ copies of $N_{\Gamma}$ is diffeomorphic to the standard sphere. By 7.3 we can make $\sharp n N_{\Gamma}$ symplectic with contact type boundary. The obstruction to the homotopical standardness of the constructed contact structure is an element of the group $\pi_{2 k-1}\left(S O_{2 k} / U_{k}\right)$. But this group is finite if $k$ is odd. Therefore, for some integer $m>0$, the manifold $\sharp m n N_{\Gamma}$ has a homotopically standard contact structure on its boundary sphere. The manifold $\sharp m n N \Gamma$ is $(k-1)$-connected but has nontrivial $k$ th homology group. 


\section{Remarks}

Theorem 6.1 raises the following question:

What contact manifolds define (up to blowing up) the topology of symplectic manifolds which they bound?

P. Kronheimer constructed an example of a contact manifold which bounds nondiffeomorphic symplectic manifolds. Such a situation arises each time when we consider the link of an isolated complex singularity which has the reducible space of deformations.

Note that links of isolated complex singularities provide interesting examples of $s$-fillable contact manifolds. T. Januszkiewicz pointed out that in the proof of 7.1, it is possible to use deformations of some Brieskorn singularities instead of plumbing constructions.

We will show in the paper [3] that for $k=2$, the assertion of 6.1 remains true without any assumption about the contact structure on the boundary sphere.

\section{References}

[1] D. Bennequin, Entrelacements et equations de Pfaff, Astérisque 107-108 (1983) 83-161.

[2] Y. Eliashberg, Classification of overtwisted contact structures on 3-manifolds, Invent. Math. 98 (1989) 623-637.

[3] __ Filling by holomorphic discs and its applications, preprint, 1989, to appear in Proc. Internat. Conf. in Low-Dimensional Topology, Durham.

[4] _ _ Topological characterization of Stein manifolds of dimension > 2, Intern. J. Math. 1 (1990).

[5] M. Gromov, Pseudo-holomorphic curves in symplectic manifolds, Invent. Math. 82 (1985) 307-347.

[6] D. McDuff, Asymptotically flat symplectic manifolds, preprint, 1989.

[7] C. Meckert, Formes de contact sur la somme connexe de deux variétés de contact, Publication de l'I.R.M.A., Strasbourg, 1980:

[8] J. Milnor, Differentiable manifolds which are homotopy spheres, Princeton University Press, Princeton, NJ, 1958.

[9] A. Weinstein, On the hypotheses of Rabinowitz's periodic orbits theorems, J. Differential Equations 33 (1979) 353-358.

Mathematical Sciences Research Institute STANFORD UNIVERSITY 